\title{
Response to Valganciclovir in Chronic Fatigue Syndrome Patients With Human Herpesvirus 6 and Epstein-Barr Virus IgG Antibody Titers
}

\author{
Tessa Watt, ${ }^{1}$ Stephanie Oberfoell, ${ }^{1}$ Raymond Balise, ${ }^{2}$ Mitchell R. Lunn, ${ }^{3}$ Aroop K. Kar, ${ }^{1}$ \\ Lindsey Merrihew, ${ }^{1}$ Munveer S. Bhangoo, ${ }^{1}$ and José G. Montoya ${ }^{1,2,4 *}$ \\ ${ }^{1}$ Division of Infectious Diseases and Geographic Medicine, Department of Medicine, \\ Stanford University Medical Center, Palo Alto, California \\ ${ }^{2}$ Stanford University School of Medicine, Health Research and Policy, Stanford, California \\ ${ }^{3}$ Stanford University School of Medicine, Stanford, California \\ ${ }^{4}$ Palo Alto Medical Foundation, Research Institute, Palo Alto, California
}

Valganciclovir has been reported to improve physical and cognitive symptoms in patients with chronic fatigue syndrome (CFS) with elevated human herpesvirus 6 (HHV-6) and Epstein-Barr virus (EBV) IgG antibody titers. This study investigated whether antibody titers against HHV-6 and EBV were associated with clinical response to valganciclovir in a subset of CFS patients. An uncontrolled, unblinded retrospective chart review was performed on 61 CFS patients treated with $900 \mathrm{mg}$ valganciclovir daily (55 of whom took an induction dose of $1,800 \mathrm{mg}$ daily for the first 3 weeks). Antibody titers were considered high if HHV-6 IgG $\geq 1: 320$, EBV viral capsid antigen (VCA) $\lg G \geq 1: 640$, and EBV early antigen (EA) IgG $\geq 1: 160$. Patients selfrated physical and cognitive functioning as a percentage of their functioning prior to illness. Patients were categorized as responders if they experienced at least $30 \%$ improvement in physical and/or cognitive functioning. Thirty-two patients (52\%) were categorized as responders. Among these, 19 patients $(59 \%)$ responded physically and 26 patients (81\%) responded cognitively. Baseline antibody titers showed no significant association with response. After treatment, the average change in physical and cognitive functioning levels for all patients was $+19 \%$ and $+23 \%$, respectively $(P<0.0001)$. Longer treatment was associated with improved response $(P=0.0002)$. No significant difference was found between responders and non-responders among other variables analyzed. Valganciclovir treatment, independent of the baseline antibody titers, was associated with self-rated improvement in physical and cognitive functioning for CFS patients who had positive HHV-6 and/or EBV serologies. Longer valganciclovir treatment correlated with an improved response. J. Med. Virol. 84:1967-

1974, 2012. ㄷ 2012 Wiley Periodicals, Inc.

KEY WORDS: CFS; HHV-6; EBV

\section{INTRODUCTION}

Chronic fatigue syndrome (CFS) is a controversial, multi-systemic disease that affects more than 1 million Americans [CDC, 2012], resulting in $\$ 9.1$ billion in lost productivity annually [Reynolds et al., 2004]. CFS is characterized clinically by severe disabling fatigue and a constellation of symptoms that

Author contributions: T.W., S.O., A.K.K., and J.G.M. designed the study. M.R.L. and J.G.M. provided patient care. T.W., S.O., A.K.K, and M.B. coordinated the study and collected the data. T.W., S.O., and R.B. performed statistical analysis and analyzed the data. T.W., S.O., R.B., M.R.L., L.M., and J.G.M. wrote the manuscript. J.G.M. supervised the project.

All authors have no financial or other conflict of interest.

Tessa Watt and Aroop K. Kar present address is 5 University of Michigan Medical School, 1301 Catherine Road, Ann Arbor, MI 48109 .

Stephanie Oberfoell present address is Los Angeles David Geffen School of Medicine, University of California, 1633 Bruin Street, Los Angeles, CA 90047.

Mitchell R. Lunn present address is Department of Medicine, Brigham and Women's Hospital, 75 Francis Street, Boston, MA 02115; Harvard Medical School, 25 Shattuck Street, Boston, MA 02115 .

Munveer S. Bhangoo present address is San Diego School of Medicine, University of California, 8950 Villa La Jolla Drive, San Diego, CA 92037.

*Correspondence to: José G. Montoya, Stanford University School of Medicine, 300 Pasteur Drive, Room S-101, Stanford, CA 94305. E-mail: gilberto@ stanford.edu

Accepted 26 July 2012

DOI 10.1002/jmv.23411

Published online in Wiley Online Library

(wileyonlinelibrary.com). 
prominently features impaired concentration and short-term memory, sleep disturbances, and musculoskeletal pain. No pathognomonic signs or diagnostic tests have been validated, and no definitive treatments are available.

CFS is recognized as a complex clinical syndrome with multiple presentations [Jason et al., 2005]. Proposed etiologies include viral or bacterial infections, environmental factors, endocrine and metabolic dysfunction, immunological imbalance, neural-mediated hypotension, and depression [Fukuda et al., 1994; Afari and Buchwald, 2003]. A viral trigger has been suspected when patients developed acute CFS following severe influenza-like symptoms, infectious mononucleosis, Q fever, or Ross River virus infection [Komaroff and Buchwald, 1998; Hickie et al., 2006]. Human herpesvirus 6 (order Herpesvirales, family Herpesviridae, subfamily Betaherpesvirinae, genus Roselolvirus, species Human herpesvirus 6; HHV-6) and Epstein-Barr virus (order Herpesvirales, family Herpesviridae, subfamily Gammaherpesvirinae, genus Lymphocryptovirus, species Human herpesvirus 4; EBV or HHV-4) are among the viruses that have been frequently associated with CFS [Komaroff, 1988, 2006; Kogelnik et al., 2006].

EBV and HHV-6 are lymphotrophic and produce latent infections with immunomodulatory effects [Ambinder, 2003; Krueger and Ablashi, 2003; Ambinder and Lin, 2005] In addition, HHV-6 has been found to be neurotropic [Ablashi et al., 2010; Flamand et al., 2010; Yao et al., 2010] and can reside in neurons [Fotheringham et al., 2008] and cardiac myocytes [Kuhl et al., 2005; Leveque et al., 2011] in a semi-active state without evidence of continued infection in the plasma compartment. HHV-6 has been associated with cognitive decline in hematopoietic stem cell transplantation, and it has been reported to cause encephalitis in 8-14\% of umbilical cord blood transplant patients [Mori et al., 2010; Hill et al., 2012]. While in vitro co-infection with both viruses has revealed significant interaction [Flamand et al., 1993; Cuomo et al., 1998], the clinical consequences of ongoing and active infections with both EBV and HHV-6 remain unknown. Co-infection with these viruses or other infectious agents may contribute to the pathogenesis of certain diseases including CFS [Komaroff, 1988; Sairenji et al., 1995] and multiple sclerosis [Hollsberg et al., 2005].

Acute EBV infection and prevalence is usually assessed by detection of antibodies against three different antigens: viral capsid antigen (VCA), nuclear antigen (NA), and early antigen (EA). EBV PCR testing is also available to support the diagnosis of acute infection or reactivation. For HHV-6, IgG, and IgM antibodies may be used to establish whether an individual is acutely or chronically infected. HHV-6 PCR DNA testing is available to support further acute infection, reactivation, or chromosomally integrated virus in select patients. IgM antibodies are inappropriate to diagnose reactivation as their levels wane within months of the primary infection and do not change or reappear with reactivation. Unfortunately, the presence of IgG does not distinguish viral reactivation from primary infection. Thus, for the suspicion of reactivation, an increase of or "high" IgG titers can only be used as an indirect evidence of reactivation. This makes identifying patients with reactivated $\mathrm{EBV}$ or $\mathrm{HHV}-6$ infection challenging.

The seroprevalence for EBV and HHV-6 is $90 \%$ or greater in the adult population, and most individuals undergo asymptomatic seroconversion. This high seroprevalence complicates the interpretation of serological tests in diagnosing reactivation and emphasizes the importance of using supplementary laboratory-based diagnostic tools to detect reactivation. While plasma DNA is a valid marker for reactivation in immunosuppressed (e.g., transplant) patients, it is unknown if reactivated infection in immunocompetent individuals may be present with detectable plasma nucleic acid levels [Leveque et al., 2011]. In healthy, EBV-seropositive subjects, humoral immunity has been demonstrated to remain stable over time with episodic reactivation occurring at a low rate [Amanna et al., 2007].

Valganciclovir has been reported to be effective clinically for treating CFS patients with positive serologies for HHV-6, EBV, and/or cytomegalovirus (CMV). Seventy-five percent of patients positive for these viruses who were treated with valacyclovir and/or valganciclovir for at least 6 months improved by $\geq 1$ point on the Energy Index Point Score (EIPS), representing a significant change in functioning [Lerner et al., 2010].

Twelve patients who were infected chronically with HHV-6 and EBV and had elevated HHV-6 and EBV antibody titers (median titers: EBV VCA IgG 1:2,560; HHV-6 IgG 1:1,280) and suffered from debilitating fatigue for at least 1 year were reported previously to experience significant improvement in their fatigue and cognitive symptoms following the open-label administration of valganciclovir for 24 weeks [Kogelnik et al., 2006]. High titers were postulated to indicate active viral replication and possibly an abnormal immune response.

In this study, a retrospective chart review of 61 patients who met the CFS case definition [Fukuda et al., 1994] was performed in which laboratory results, patient self-reported data, and clinical observations were analyzed to determine whether antibody titers to HHV-6 IgG, EBV VCA IgG, EBV NA IgG, and EBV EA IgG were associated with response to valganciclovir treatment. Patients with high baseline antibody titers (HHV-6 IgG $\geq 1: 320$, EBV VCA IgG $\geq 1: 640$, and EBV EA IgG $\geq 1: 160$ ) were hypothesized to be more likely to respond. Secondary and exploratory analyses of physical and cognitive functioning levels, change in antibody titers with treatment, age at treatment, fatigue duration, and treatment duration were conducted.

\section{MATERIALS AND METHODS}

\section{Patient Selection}

A retrospective chart review was performed on all records of 70 CFS patients who had been treated with 
valganciclovir at Stanford University Medical Center between February 2004 and January 2009. The inclusion criteria were: (i) meeting the CDC CFS case definition [Fukuda et al., 1994], (ii) prior valganciclovir treatment, and (iii) complete records for self-reported functioning levels and antibody titers. Complete records required baseline and final cognitive and physical functioning levels as well as baseline and final antibody titers for HHV-6 IgG, EBV VCA IgG, EBV NA IgG, and EBV EA IgG. A total of 64 patients met these criteria. Of this total, six participants did not have final titer measurements; however, these patients were included because their data were complete for the primary endpoint. As a result, the study excluded these six patients in the secondary analyses involving final titers and change in titer (Fig. 1). Three of the 64 patients in this analysis were included in a prior study [Kogelnik et al., 2006], and were therefore excluded, leaving a final sample size of 61 . Patient information was obtained from chart records and direct patient inquiry. Patient demographics (e.g., ethnicity, sex, age) were not used for inclusion.

\section{Valganciclovir Treatment}

Patients followed one of two valganciclovir treatment regimens based on clinical judgment (e.g., history of side effects to antimicrobials). Fifty-eight patients took $900 \mathrm{mg}$ of valganciclovir twice daily (induction dose) for 3 weeks, followed by $900 \mathrm{mg}$ of valganciclovir once daily (maintenance dose). Six patients only took the maintenance dose for the duration of their treatment.

The length of treatment varied among patients according to the treating physician's clinical judgment. If patients were treated with valganciclovir more than once and the two treatment regimens were more than 1 month apart, then the first treatment

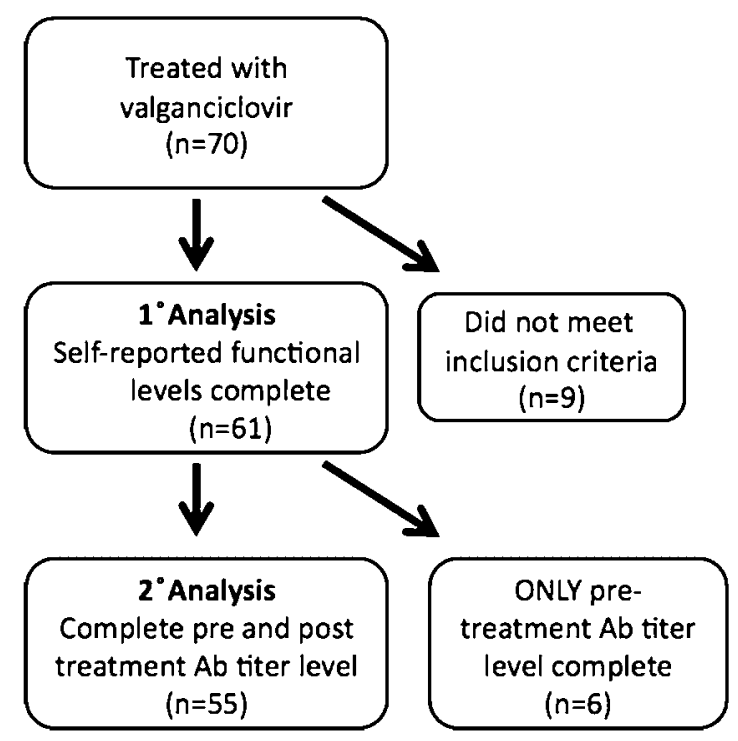

Fig. 1. Patient flow. The number of patients that met the inclusion criteria for primary and secondary analyses. regimen was used for analysis as well as for establishing baseline and final functioning levels and antibody titers. If, however, multiple treatments with valganciclovir were $<1$ month apart, these were considered a single treatment course for analysis purposes.

\section{EBV and HHV-6 Antibody Titers}

Baseline and final titers were recorded for HHV-6 IgG, EBV VCA IgG, EBV NA IgG, and EBV EA IgG before and after treatment with valganciclovir. To limit variation introduced by using multiple laboratories, all antibody titers were measured by using an immunofluorescent antibody (IFA)-based assay performed by Focus Diagnostics (Cypress, CA). Titers were reported as reciprocal dilutions. Since the distribution showed marked skew, titers were natural logtransformed to allow for statistical comparison between dilutions (Appendix 1). For sensitivity analyses, non-parametric Wilcoxon-signed rank tests were conducted.

Baseline antibody titers were defined as the highest titer within 1 year prior to starting treatment. The highest titers prior to treatment (rather than the average of all available titers) were chosen because high titers of a certain threshold were considered to have the greatest pathological impact and because EBV titers have been shown to be remarkably stable over long-term follow-up of healthy individuals [Amanna et al., 2007]. Final titers were defined as those closest to the cessation of treatment but no later than 6 months after stopping treatment. If no such value existed, final titers were defined to be those closest to the end of treatment up to 1 month prior to stopping treatment.

For qualitative analysis, baseline antibody titers were categorized as high or low. The threshold for high baseline titers was defined as HHV-6 IgG $\geq 1: 320$, EBV VCA IgG $\geq 1: 640$, and EBV EA IgG $\geq 1: 160$. All three antibodies were required to meet these defined thresholds. These criteria were chosen based on the authors' clinical experience with evaluating antibody titers of CFS patients. Since prior research has not explored the clinical significance for EBV NA IgG titers in CFS patients, EBV NA IgG titers were not used for assigning baseline titer category but were still included in analyses to explore potential significance.

\section{Physical and Cognitive Functioning}

To assess response to treatment, self-reported physical and cognitive functioning levels were obtained at every clinic visit. Functioning levels were evaluated on a scale of $0-100 \%$. A score of $100 \%$ indicated that the patient was completely well.

Baseline physical and cognitive functioning levels were determined by averaging up to three levels within 1 year prior to starting valganciclovir treatment. Final functioning levels were determined by identifying the highest level within 6 months of stopping 
treatment. The highest levels after treatment (rather than the average of all available levels) were chosen in order to capture the largest effect of treatment on functioning. Additionally, a wide variation in time to treatment benefit was observed (e.g., functioning may improve during treatment course or after treatment cessation). The average of post-treatment functioning levels, therefore, may not present an accurate depiction of improvement.

For qualitative analyses, a responder was defined as a patient with a self-reported physical or cognitive functioning increase after treatment of at least $30 \%$. To explore the distinction between those who respond physically and those who respond cognitively, responders were further categorized as physical and/or cognitive responders (i.e., physical responders reported at least 30\% improvement in physical functioning, cognitive responders reported at least 30\% improvement in cognitive functioning). Non-responders improved $<30 \%$ physically and cognitively. Quantitative analysis was performed on change in physical and cognitive functioning levels independent of response category (responder/non-responder).

\section{Statistical Methods}

Three primary analyses were conducted. The first assessed the relationship between baseline antibody titers and response to treatment. For this, four unadjusted logistic regression models using natural logtransformed antibody titers for HHV-6 IgG, EBV VCA IgG, EBV NA IgG, and EBV EA IgG were used to predict response to valganciclovir treatment. For each of these predictors, an adjusted model was also built to control for sex, age at treatment, and fatigue duration. The secondary analysis used paired $t$-tests to assess change in mean self-reported physical and cognitive functioning with valganciclovir treatment. As a tertiary exploratory analysis, differences in mean antibody titer levels before and after treatment were compared with one-sample $t$-tests with a null hypothesis of no change. Two-sample $t$-tests were used to assess the amount of change in each antibody titer between responders and non-responders.

Additional analysis, such as contrasts in demographics, used one or two-sample $t$-tests or chi-square tests as appropriate. Statistical significance on all tests was set at $P<0.05$. All statistical analyses were performed using SAS version 9.2 with SAS Enterprise Guide version 4.22 on Microsoft Windows XP.

\section{Ethics Approval}

This study was reviewed and approved by the Stanford University Institutional Review Board. Exemption for informed consent was granted given the retrospective, chart review study design. All components of this study were conducted in compliance with local, state, and federal laws, institutional regulations and policies, and the ethical standards of the Declaration of Helsinki.

\section{RESULTS}

\section{Baseline Characteristics}

The CFS patients studied consisted of 42 females (69\%) and 19 males (31\%). The subjects varied widely in age, duration of fatigue prior to valganciclovir treatment, and length of treatment (Table I).

Thirty-five patients (57\%) were considered to have high baseline antibody titers. Older age $(P=0.0323)$ and longer fatigue duration $(P=0.0364)$ were associated with higher baseline titers (Table I). No significant difference was found between baseline titer category with respect to sex.

\section{Association of Baseline Titers and Response}

Thirty-two patients (52\%) were categorized as responders. Of the responders, 19 patients (59\%) responded physically and 26 patients $(81 \%)$ responded cognitively. Neither unadjusted nor adjusted logistic regression of the natural $\log$ of baseline antibody

TABLE I. Patient Characteristics and Effect of Valganciclovir Treatment on Physical and Cognitive Functioning by Antibody Titer Group

\begin{tabular}{|c|c|c|c|c|c|}
\hline & $\begin{array}{l}\text { All patients }(\mathrm{n}=61) \\
\text { mean }(\mathrm{SD})\end{array}$ & $P$-value ${ }^{\mathrm{a}}$ & $\begin{array}{l}\text { High titers }(\mathrm{n}=35), \\
\text { mean }(\mathrm{SD})\end{array}$ & $\begin{array}{l}\text { Low titers }(\mathrm{n}=26), \\
\text { mean }(\mathrm{SD})\end{array}$ & $P$-value ${ }^{\mathrm{b}}$ \\
\hline Age at treatment, year & $47(14)$ & & $51(13)$ & $43(15)$ & 0.0323 \\
\hline Fatigue duration, year & $12(10)$ & & $14(12)$ & $8.8(7.5)$ & 0.0364 \\
\hline Treatment length, month & $6.5(2.0)$ & & $6.7(2.2)$ & $6.2(1.9)$ & NS \\
\hline All responders, \% & 52 & & 62 & 41 & NS \\
\hline Physical responders, \% & 31 & & 32 & 33 & NS \\
\hline Cognitive responders, $\%$ & 43 & & 51 & 33 & NS \\
\hline Physical functioning change, $\%$ & $+19(22)$ & $<0.0001$ & $+20(21)$ & $+18(23)$ & NS \\
\hline Cognitive functioning change, $\%$ & $+23(21)$ & $<0.0001$ & $+26(19)$ & $+19(23)$ & NS \\
\hline
\end{tabular}

SD, standard deviation; NS, not significant.

aThis $P$-value refers to the significance of the change in physical and cognitive functioning after treatment for all patients.

${ }^{b}$ This $P$-value refers to the significance of the difference in the indicated values between patients in the high titer group and the low titer group. 
titers was statistically associated with response to treatment. An analogous analysis showed that there was no significant difference in mean baseline antibody titers between responders and non-responders (Table II). There was no significant difference in mean change of physical and cognitive functioning between high and low baseline antibody titer categories (Table I).

\section{Changes in Physical and Cognitive Functioning}

Before valganciclovir treatment, the average baseline physical and cognitive functioning levels were $34 \%$ (standard deviation; SD 6.9\%) and $43 \%$ (SD $5.4 \%$ ), respectively, for all patients (responders and non-responders). After treatment, the mean of all patients' physical and cognitive functioning levels improved by an average of $19 \%(P<0.0001)$ and $23 \%$ $(P<0.0001)$, respectively (Table I, Fig. 2a-c). The mean change in physical and cognitive functioning levels was $+34 \%$ (SD 19\%) and $+37 \%$ (SD 18\%), respectively, in the responder group and $+3.1 \%$ (SD $10 \%$ ) and $+7.7 \%$ (SD $10 \%$ ), respectively, in the nonresponder group.

\section{Change in Titers}

Mean antibody titers for EBV VCA IgG $(P=0.0165)$ and HHV-6 IgG $(P<0.0001)$ decreased with valganciclovir treatment in all patients (responders and non-responders). No significant difference was found in the change of antibody titers after treatment between the responder and non-responder groups (Table II).

\section{Responder Status}

No significant difference was found between responders and non-responders with respect to sex and baseline titer category (high/low). However, longer treatment length was associated with the responder group $(P=0.0002)$. There was no significant difference between responders and non-responders with regard to age at treatment and fatigue duration (Table II).

\section{DISCUSSION}

The patients in this study had a mean age of 47 years at the time of treatment and $70 \%$ were female, an age and sex distribution representative of the larger CFS population in the United States [Jason et al., 1999]. All patients improved by a mean $19 \%$ and $23 \%$ in physical and cognitive functioning, respectively, after valganciclovir treatment $(P<0.0001)$. For responders, the mean physical and cognitive improvement was $34 \%$ and $37 \%$, respectively. The standard deviation of patients' baseline physical and cognitive functioning levels was notably very low compared to the significant mean improvement in functioning levels after treatment, emphasizing the significant recovery. It was, however, not possible to assess the possibility of placebo or Hawthorn effects due to the single-arm, historical study design.

A 2010 study used a different validated scale for measuring severity of fatigue, the Energy Index Point Score (EIPS) [Lerner et al., 2008]. The EIPS ranks fatigue on a scale from 0 to 10 , and an improvement of at least 1 point qualifies a patient as a responder [Lerner et al., 2010]. In comparing this study's physical and cognitive functioning scale to the EIPS, a change of 1 point out of 10 would be loosely comparable to a $10 \%$ improvement. However, this study categorized only those patients whose physical or cognitive functioning improved at least $30 \%$ as responders. If this study had used a similar responder definition, the change would have yielded a much higher proportion of responders than non-responders than is currently reported in this study. In the context

TABLE II. Patient Characteristics, Baseline Physical and Cognitive Functioning and Antibody Titers, and Change in Physical and Cognitive Functioning and Antibody Titers After Valganciclovir Treatment by Responder Group

\begin{tabular}{|c|c|c|c|c|c|c|c|}
\hline & $\begin{array}{l}\text { All patients } \\
(\mathrm{n}=61) \\
\text { mean }(\mathrm{SD})\end{array}$ & $\mathrm{P}$-value $\mathrm{a}^{\mathrm{a}}$ & $\begin{array}{l}\text { Responders } \\
\quad(\mathrm{n}=32), \\
\text { mean }(\mathrm{SD})\end{array}$ & $\begin{array}{l}\text { Non-responders } \\
\qquad(\mathrm{n}=29) \\
\text { mean (SD) }\end{array}$ & $\mathrm{P}$-value $\mathrm{e}^{\mathrm{b}}$ & $\begin{array}{l}\text { Unadjusted } \\
\text { OR (CL) }\end{array}$ & $\begin{array}{l}\text { Adjusted } \\
\text { OR (CL) }\end{array}$ \\
\hline Age at treatment, year & $47(14)$ & & $49(12)$ & $45(16)$ & NS & & \\
\hline Fatigue duration, year & $12(10)$ & & $12(11)$ & $12(9.4)$ & NS & & \\
\hline Treatment length, month & $6.5(2.0)$ & & $7.4(2.4)$ & $5.5(0.9)$ & 0.0002 & & \\
\hline Baseline EBV VCA & $7.7(2.0)$ & & $7.9(1.3)$ & $7.4(2.5)$ & NS & $1.1(0.86-1.5)$ & $1.1(0.81-1.5)$ \\
\hline Baseline EBV NA & $5.1(1.5)$ & & $5.1(1.2)$ & $4.9(1.8)$ & NS & $1.1(0.77-1.5)$ & $1.0(0.72-1.5)$ \\
\hline Baseline EBV EA & $5.6(1.5)$ & & $5.7(1.1)$ & $5.4(1.8)$ & NS & $1.2(0.81-1.6)$ & $1.1(0.78-1.7)$ \\
\hline Baseline HHV-6 & $6.5(1.5)$ & & $6.5(1.3)$ & $6.6(1.6)$ & NS & $0.96(0.68-1.4)$ & $0.86(0.59-1.3)$ \\
\hline EBV VCA change & $-0.47(1.4)$ & 0.0165 & $-0.67(1.1)$ & $-0.26(1.7)$ & NS & & \\
\hline EBV NA change & $-0.18(1.3)$ & NS & $-0.13(1.3)$ & $-0.24(1.3)$ & NS & & \\
\hline EBV EA change & $-0.29(1.1)$ & NS & $-0.49(1.0)$ & $-0.12(1.1)$ & NS & & \\
\hline HHV- 6 change & $-0.86(1.2)$ & $<0.0001$ & $-0.67(1.4)$ & $-1.1(0.97)$ & NS & & \\
\hline
\end{tabular}

$\mathrm{SD}$, standard deviation; OR, odds ratio; CL, confidence limit; NS, not significant.

aThis $P$-value refers to the significance of the change in titers after treatment for all patients.

b This $P$-value refers to the significance of the difference in the indicated values between responders and non-responders. 
of the literature and given the difficulty that most CFS patients have attaining any level of recovery, these findings are clinically striking and suggest that valganciclovir treatment may result in substantial improvement in physical and/or cognitive functioning in CFS patients with positive HHV-6 and EBV antibody titers.

In contrast to this study's original hypotheses, there was no statistically significant association between
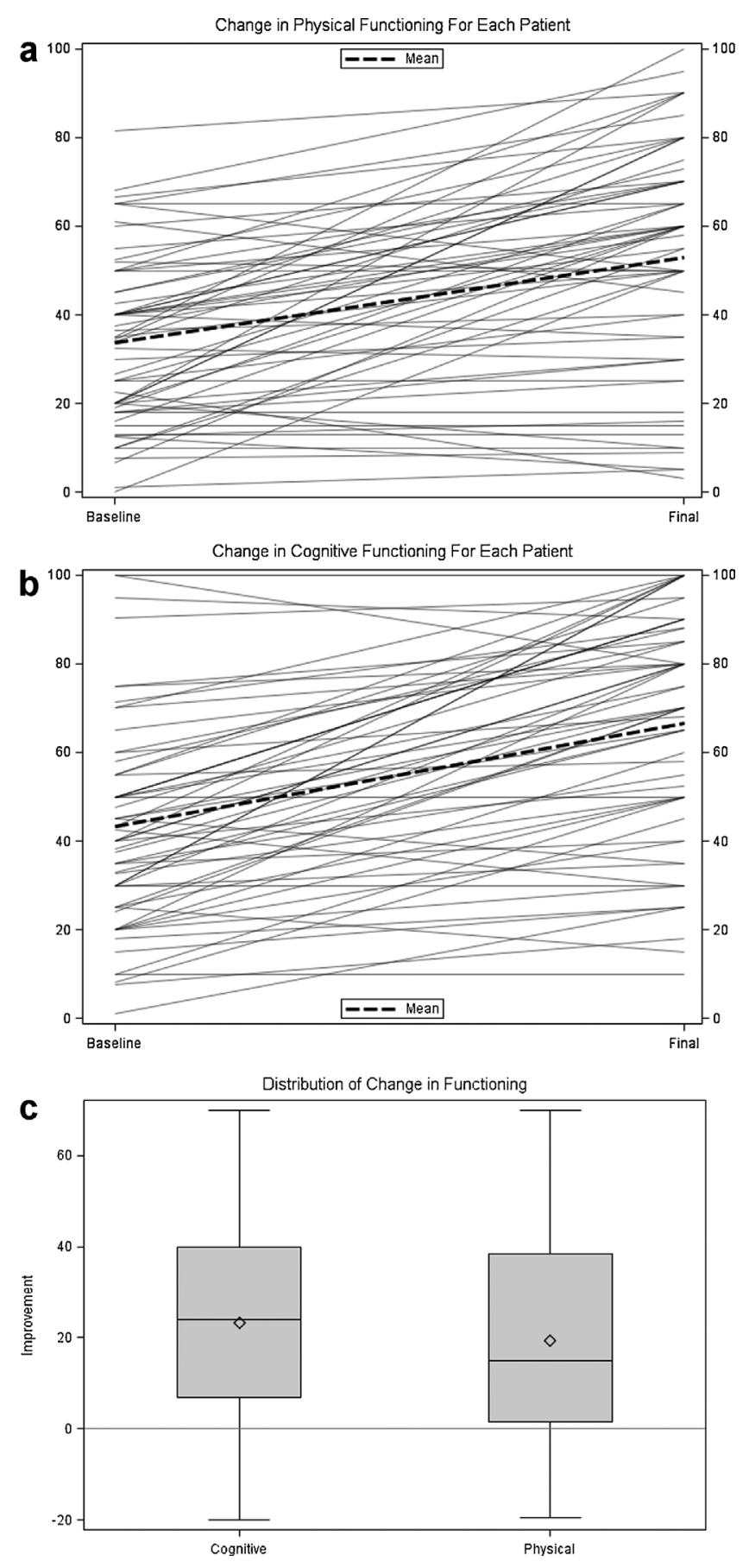

baseline antibody titers and response to valganciclovir treatment. The high baseline titer group exhibited a trend toward increased physical and cognitive improvement. Fifty-one percent $(51 \%)$ of patients with high titers were cognitive responders compared to only $33 \%$ of those with low titers. Based on these trends, higher baseline EBV titers may still correlate with improved response to valganciclovir. Furthermore, after completion of this study an evaluation of 50 healthy individuals was found to have a median EBV VCA IgG 1:640, EBV EA IgG 1:160, and HHV-6 IgG 1:160 (personal communication from Focus Diagnostics, Inc. to J.G.M.) The pure effect of baseline antibody titer on clinical improvement or the modest magnitude of baseline antibody titers in this study may not be large enough to detect an effect with this sample size, as individual antibody titers can vary by two dilutions under normal circumstances. Furthermore, some patients with reactivated virus may have borderline hypogammaglobulinemia. In these patients, a low viral IgG may actually represent a significant relative proportion of total IgG. Conversely, patients with notably strong immune systems may have more robust serological responses. Larger, prospective studies are needed to substantiate the small effect observed in this study and to look for non-linearities (e.g., thresholds) in antibody titer level that may predict response to treatment.

Valganciclovir treatment caused all mean antibody titers to decrease in responders and non-responders. The decline in both EBV VCA and HHV-6 antibody titers observed in this open-label cohort suggests that our patients might have had a substantial reactivation that was amenable to antiviral intervention against both viruses. The decline in EBV VCA and EBV EA antibody titers was more pronounced in responders than non-responders; however, this distinction was not statistically significant. This study was not powered to detect significant differences in the mean change between these groups.

With regard to age at treatment and fatigue duration, there was no significant difference between responders and non-responders. However, longer treatment duration was associated with improved responder status, which suggests that longer treatment

Fig. 2. a: Change in physical functioning for each patient $(n=61)$. Change in each patient's physical functioning on a $0-100 \%$ scale from baseline to end of valganciclovir treatment. Each solid line represents one patient. Dashed line indicates mean change over treatment period. b: Change in cognitive functioning for each patient $(\mathrm{n}=61)$. Change in each patient's cognitive functioning on a $0-100 \%$ scale from baseline to end of valganciclovir treatment. Each solid line represents one patient. Dashed line indicates mean change over treatment period. c: Distribution of change in functioning $(n=61)$. Distribution of change in physical and cognitive functioning on a 0 $100 \%$ scale after valganciclovir treatment for all patients. Within each box, the diamond represents the mean and the line indicates the median change in functioning level. The lower limit of each box indicates the 25 th percentile and the upper limit indicates the 75 th percentile of change in functioning level. The lower whisker outside each box indicates the minimum change in functioning level and the upper whisker indicates the maximum change in functioning level. 
may improve the likelihood of responding to valganciclovir. Decisions on treatment duration were made on an individual basis between the physician and patient, which may bias the relationship between treatment duration and response. Future randomization of patients to different treatment durations is needed to confirm these findings and to assess if there is a linear duration-response correlation. CFS patients with herpesvirus infections on antiviral medication continue to experience benefit for at least 2-3 years while on treatment [Lerner et al., 2010].

This study has several limitations. Because it is a retrospective study, this report has the limitation of excluding nine patients who did not meet the inclusion criteria. The absence of a control population in this study makes it impossible to draw definitive conclusions regarding treatment effect. Prospective, randomized, double-blind, placebo-controlled studies are needed to explore the clinical relevance of these findings.

While self-report assessments are not an ideal tool for evaluating CFS patients' functioning levels, the authors' research and clinical experience have demonstrated that this system of self-reported assessments is an informative measure of physical and cognitive functioning. Though self-reported baseline and final functioning levels may have inter-rater variability, this study addressed this problem by evaluating intra-rater change in functioning and contrasted only the inter-rater magnitude of change to evaluate treatment efficacy.

In the context of current research, the magnitude of physical and cognitive improvement after valganciclovir treatment was substantial. These findings emphasize the importance of developing tools to select candidates for valganciclovir treatment who are likely to respond. Identifying factors predictive of response prior to treatment initiation will enable providers to prescribe valganciclovir or other antiviral interventions effectively to CFS patients. While this study was underpowered to conduct hypothesis testing on the differences between responders and nonresponders, it suggests that valganciclovir can be associated with self-rated improvement in physical and cognitive functioning in a subset of CFS patients.

\section{ACKNOWLEDGMENTS}

We would like to thank the patients in the Stanford CFS Clinic for their patience and willingness to be part of efforts to enhance the understanding and treatment of this unexplained illness. The authors also thank Manisha Desai, Ph.D. for her critical review of the article and thoughtful suggestions for statistical analysis. M.R.L. was supported by the Stanford University School of Medicine Molecular Basis of Medicine Scholarly Concentration and the Stanford University School of Medicine Medical Scholars Fellowship Program.

\section{APPENDIX 1}

TABLE A1. Natural Log (LN) Transformation of EBV and HHV-6 IgG Antibody Titers

\begin{tabular}{lcccc}
\hline EBV VCA & EBV NA & EBV EA & HHV-6 & LN \\
\hline $1: 10$ & $1: 10$ & $1: 10$ & $1: 10$ & 2.30 \\
$1: 20$ & $1: 20$ & $1: 20$ & $1: 20$ & 3.00 \\
$1: 40$ & $1: 40$ & $1: 40$ & $1: 40$ & 3.69 \\
$1: 80$ & $1: 80$ & $1: 80$ & $1: 80$ & 4.38 \\
$1: 160$ & $1: 160$ & $1: 160$ & $1: 160$ & 5.08 \\
$1: 320$ & $1: 320$ & $1: 320$ & $1: 320$ & 5.77 \\
$1: 640$ & $1: 640$ & $1: 640$ & $1: 640$ & 6.46 \\
$1: 1,280$ & $1:>640^{\mathrm{a}}$ & $1:>640^{\mathrm{a}}$ & $1: 1,280$ & 7.15 \\
$1: 2,560$ & & & $1: 2,560$ & 7.85 \\
$1: 5,120$ & & & $1: 5,120$ & 8.54 \\
$1: 10,240$ & & & $1: 10,240$ & 9.23 \\
$1:>10,240^{\mathrm{a}}$ & & & $1:>10,240^{\mathrm{a}}$ & 9.93 \\
\hline
\end{tabular}

aThese values were rounded up to the next dilution to facilitate quantitative analysis.

\section{REFERENCES}

Ablashi DV, Devin CL, Yoshikawa T, Lautenschlager I, Luppi M, Kuhl U, Komaroff AL. 2010. Review Part 3: Human herpesvirus6 in multiple non-neurological diseases. J Med Virol 82: 1903-1910.

Afari N, Buchwald D. 2003. Chronic fatigue syndrome: A review. Am J Psychiat 160:221-236.

Amanna IJ, Carlson NE, Slifka MK. 2007. Duration of humoral immunity to common viral and vaccine antigens. N Engl J Med 357:1903-1915.

Ambinder RF. 2003. Epstein-Barr virus-associated lymphoproliferative disorders. Rev Clin Exp Hematol 7:362-374.

Ambinder RF, Lin L. 2005. Mononucleosis in the laboratory. J Infect Dis 192:1503-1504.

Centers for Disease Control and Prevention (CDC). Chronic Fatigue Syndrome-Updated May 16, 2012. Available from: http://www. cdc.gov/cfs/

Cuomo L, Trivedi P, de Grazia U, Calogero A, D’Onofrio M, Yang W, Frati L, Faggioni A, Rymo L, Ragona G. 1998. Upregulation of Epstein-Barr virus-encoded latent membrane protein by human herpesvirus 6 superinfection of EBV-carrying Burkitt lymphoma cells. J Med Virol 55:219-226.

Flamand L, Stefanescu I, Ablashi DV, Menezes J. 1993. Activation of the Epstein-Barr virus replicative cycle by human herpesvirus 6 . J Virol 67:6768-6777.

Flamand L, Komaroff AL, Arbuckle JH, Medveczky PG, Ablashi DV 2010. Review, part 1: Human herpesvirus-6-basic biology, diagnostic testing, and antiviral efficacy. J Med Virol 82:1560-1568.

Fotheringham J, Williams EL, Akhyani N, Jacobson S. 2008. Human herpesvirus 6 (HHV-6) induces dysregulation of glutamate uptake and transporter expression in astrocytes. J Neuroimmune Pharm 3:105-116.

Fukuda K, Straus SE, Hickie I, Sharpe MC, Dobbins JG, Komaroff A. 1994. The chronic fatigue syndrome: A comprehensive approach to its definition and study. International Chronic Fatigue Syndrome Study Group. Ann Intern Med 121:953-959.

Hickie I, Davenport T, Wakefield D, Vollmer-Conna U, Cameron B, Vernon SD, Reeves WC, Lloyd A. 2006. Post-infective and chronic fatigue syndromes precipitated by viral and non-viral pathogens: Prospective cohort study. Brit Med J 333:575-578.

Hill JA, Koo S, Guzman Suarez BB, Ho VT, Cutler C, Koreth J, Armand P, Alyea EP III, Baden LR, Antin JH, Soiffer RJ, Marty FM. 2012. Cord-blood hematopoietic stem cell transplant confers an increased risk for human herpesvirus-6-associated acute limbic encephalitis: A cohort analysis. Biol Blood Marrow Transplant 1-11; [Epub ahead of print].

Hollsberg P, Kusk M, Bech E, Hansen HJ, Jakobsen J, Haahr S. 2005. Presence of Epstein-Barr virus and human herpesvirus $6 \mathrm{~B}$ 
DNA in multiple sclerosis patients: Associations with disease activity. Acta Neurol Scand 112:395-402.

Jason LA, Richman JA, Rademaker AW, Jordan KM, Plioplys AV Taylor RR, McCready W, Huang CF, Plioplys S. 1999. A community-based study of chronic fatigue syndrome. Arch Intern Med 159: 2129-2137.

Jason LA, Corradi K, Torres-Harding S, Taylor RR, King C. 2005 Chronic fatigue syndrome: The need for subtypes. Neuropsychol Rev 15:29-58.

Kogelnik AM, Loomis K, Hoegh-Petersen M, Rosso F, Hirschier C Montoya JG. 2006. Use of valganciclovir in patients with elevated antibody titers against Human Herpesvirus-6 (HHV-6) and Epstein-Barr Virus (EBV) who were experiencing central nervous system dysfunction including long-standing fatigue. J Clin Virol 37:S33-S38.

Komaroff AL. 1988. Chronic fatigue syndromes: Relationship to chronic viral infections. J Virol Methods 21:3-10.

Komaroff AL. 2006. Is human herpesvirus-6 a trigger for chronic fatigue syndrome? J Clin Virol 37:S39-S46.

Komaroff AL, Buchwald DS. 1998. Chronic fatigue syndrome: An update. Annu Rev Med 49:1-13.

Krueger GR, Ablashi DV. 2003. Human herpesvirus-6: A short review of its biological behavior. Intervirology 46:257-269.

Kuhl U, Pauschinger M, Seeberg B, Lassner D, Noutsias M, Poller W, Schultheiss HP. 2005. Viral persistence in the myocardium is associated with progressive cardiac dysfunction. Circulation 112: 1965-1970.
Lerner MA, Beqaj SH, Fitzgerald JT. 2008. Validation of the energy index point score to serially measure the degree of disability in patients with chronic fatigue syndrome. In Vivo 22:799-801.

Lerner MA, Beqaj SH, Fitzgerald JT, Gill K, Gill C, Edington J. 2010. Subset-directed antiviral treatment of 142 herpesvirus patients with chronic fatigue syndrome. Virus Adapt Treat $2: 11$.

Leveque N, Boulagnon C, Brasselet C, Lesaffre F, Boutolleau D, Metz D, Fornes P, Andreoletti L. 2011. A fatal case of Human Herpesvirus 6 chronic myocarditis in an immunocompetent adult. J Clin Virol 52:142-145.

Mori Y, Miyamoto T, Nagafuji K, Kamezaki K, Yamamoto A, Saito N, Kato K, Takenaka K, Iwasaki H, Harada N, Abe Y, Teshima T, Akashi K. 2010. High incidence of human herpes virus 6associated encephalitis/myelitis following a second unrelated cord blood transplantation. Biol Blood Marrow Transplant 16: 1596-1602.

Reynolds WJ, Vernon SD, Bouchery E, Reeves WC. 2004. The economic impact of chronic fatigue syndrome. Cost Eff Resour Alloc 2:4-12.

Sairenji T, Yamanishi K, Tachibana Y, Bertoni G, Kurata T. 1995. Antibody responses to Epstein-Barr virus, human herpesvirus 6 and human herpesvirus 7 in patients with chronic fatigue syndrome. Intervirology 38:269-273.

Yao K, Crawford JR, Komaroff AL, Ablashi DV, Jacobson S. 2010. Review part 2: Human herpesvirus-6 in central nervous system diseases. J Med Virol 82:1669-1678. 